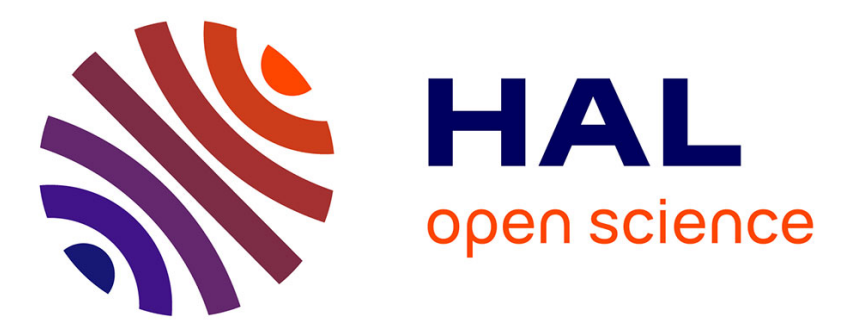

\title{
Design and testing of 3D-printed micro-architectured polymer materials exhibiting a negative Poisson's ratio
}

Filippo Agnelli, Andrei Constantinescu, Grigor Nika

\section{To cite this version:}

Filippo Agnelli, Andrei Constantinescu, Grigor Nika. Design and testing of 3D-printed microarchitectured polymer materials exhibiting a negative Poisson's ratio. Continuum Mechanics and Thermodynamics, 2019, 10.1007/s00161-019-00851-6 . hal-02410356

\section{HAL Id: hal-02410356 https://hal.science/hal-02410356}

Submitted on 6 May 2020

HAL is a multi-disciplinary open access archive for the deposit and dissemination of scientific research documents, whether they are published or not. The documents may come from teaching and research institutions in France or abroad, or from public or private research centers.
L'archive ouverte pluridisciplinaire HAL, est destinée au dépôt et à la diffusion de documents scientifiques de niveau recherche, publiés ou non, émanant des établissements d'enseignement et de recherche français ou étrangers, des laboratoires publics ou privés. 
Filippo Agnelli • Andrei Constantinescu - Grigor Nika

\title{
Design and testing of 3D-printed micro-architectured polymer materials exhibiting a negative Poisson's ratio
}

\begin{abstract}
This work proposes the complete design cycle for several auxetic materials where the cycle consists of three steps (i) the design of the micro-architecture, (ii) the manufacturing of the material and (iii) the testing of the material. We use topology optimization via a level-set method and asymptotic homogenization to obtain periodic micro-architectured materials with a prescribed effective elasticity tensor and Poisson's ratio. The space of admissible micro-architectural shapes that carries orthotropic material symmetry allows to attain shapes with an effective Poisson's ratio below -1 . Moreover, the specimens were manufactured using a commercial stereolithography Ember printer and are mechanically tested. The observed displacement and strain fields during tensile testing obtained by digital image correlation match the predictions from the finite element simulations and demonstrate the efficiency of the design cycle.
\end{abstract}

Keywords Auxetic material $\cdot$ Topology optimization $\cdot 3 \mathrm{D}$ printing $\cdot$ Polymer

\section{Introduction}

The Poisson's ratio $(v)$ is a measure of the relative amount a given material contracts transversally under a uniaxial stretch loading [17]. Unlike most conventional materials, auxetic materials tend to expand transversely to an applied uniaxial stretch load and vice versa, leading to a so-called negative Poisson's ratio. This effect occurs due to the particular internal micro-structure and due to the mechanisms of deformation when loaded. Because of their special mechanical properties, tailored auxetic materials can display enhanced stiffness and energy absorption capabilities [21,27], indentation resistance [25], greater fracture toughness [11], crashworthiness [20], phononic performance [38] as well as many other interesting properties, making them suitable in targeted applications [13,36].

Since the seminal works performed in the 80 s, the design of periodic auxetic structures has attracted research interests and several types of auxetic materials have been introduced. In 1985, Almgren [6] introduced a re-entrant honeycomb structure with Poisson's ratio of -1 using rods, hinges and springs. The re-entrant honeycomb structure was also introduced as a "bi-mode" extremal material which supports a stress with a negative determinant in Milton and Cherkaev [30]. Conceptual designs of composite materials with Poisson's ratio approaching -1 were presented in [29]. Some important features of auxetic materials, such as the reentrant corners, were discussed in the key works of Lakes [23,24], Friis et al. [15], Evans [14]. A new class of

F. Agnelli · A. Constantinescu $(\varangle)$

LMS, CNRS, École Polytechnique, Institut polytechnique de Paris, 91128 Palaiseau, France

E-mail: andrei.constantinescu@ polytechnique.edu

G. Nika

Weierstrass Institute for Applied Analysis and Stochastics, Mohrenstrasse 39, 10117 Berlin, Germany 
auxetic materials that obtain their auxetic behaviour from the rotating squares mechanism were introduced in the works of Grima et al. [18]. Moreover, a separate class of 3D auxetics that exploits the buckling mechanism in structures was proposed by [7].

Design techniques using modern numerical methods such as shape and topology optimization [1,9] arose as a natural way to tailor mechanical properties through a design of complex geometries. In auxetic material with a periodic micro-structure, the effective elastic properties can be derived by means of asymptotic homogenization, with periodic boundary conditions applied on a unit cell and the associated boundary value problem resolved $[8,28,35]$. The works of Sigmund presented a topology optimization framework for designing 2D and stacked 2D auxetic truss-based structures [37] and for designing 2D continuum-based micro-mechanism with negative Poisson's ratio [26]. Since these works, different techniques have been adopted for auxetic structure design optimization, for example SIMP, phase-field, level-set methods, etc. In the works of Wang et al. $[12,41]$, the SIMP method was used to include geometrical nonlinearities and to tackle manufacturing constraints. The resulting architectures attain the desired response through uniform features, like the thickness of connecting rods. In other recent developments, Carraturo et al. [22], Choi and Lakes [33] and Clausen et al. [10] extend this method to thermodynamic topology optimization or graded materials. Furthermore, alternative optimization methods as found in the works of Wang et al. [42,43], Vogiatzis et al. [40] and Nika and Constantinescu [31], among others, use asymptotic homogenization, the level-set method [32] and the Hadamard shape derivative to identify material regions and track boundary changes to systematically design auxetic shapes. Wang et al. [42], design linear elastic and thermoelastic materials with negative Poisson's ratio, while Nika and Constantinescu [31] design linear elastic multi-materials with negative Poisson's ratio.

The classical theory of elasticity states that isotropic three-dimensional materials may exhibit Poisson's ratios bounded in $[-1,0.5]$; Two-dimensional isotropic systems can exhibit Poisson's ratios bounded in $[-1,1]$. The effective elastic tensor that characterizes the auxetic material however has a priori orthotropic symmetry. In two-dimensional systems, the effective elastic stiffness is characterized by two Poisson ratios $v_{12}, v_{21}$, which are a priori not bounded, hence they can assume any positive or negative values in certain directions [39]. For instance, Poisson's ratios smaller than -1 have been reported according to Lakes [24]. In the topology optimization literature, the auxetic shapes obtained tend to exhibit cubic symmetry, i.e. $v_{12}=v_{21}$. However, as was already mentioned, this need not be the case.

The aim of this paper is to complete a design cycle for several auxetic materials. We combine topology optimization to systematically obtain the micro-architecture with 3D printing to digitally fabricate the designs and validate against the numerically predicted behaviour. Materials are manufactured using a desktop stereolithography 3D printer and then tested on standard tensile machine. Insight into the local mechanical fields is obtained using digital image correlation. The paper is organized as follows. Section 2 presents the computational design of the micro-architectured material. It provides some basic results needed from the theory of homogenization, relates the effective coefficients to the effective Poisson's ratio, and sets up the optimization problem to systematically identify optimal auxetic shapes. Section 3 presents the optimal auxetic micro-structures obtained and describes some of their properties as well as the additive manufacturing process. Section 4 is dedicated to the experimental testing of the structures and the interpretation of results using digital image correlation (DIC). Additionally, an appendix reviews the approach used to measure the effective Poisson's ratio by DIC and the finite element method on periodic structures.

Notation Scalars are denoted by italic letters, $\alpha$, vectors and second-order tensors are denoted by bold face letters $\mathbf{u}$, their use being clear from the context of the equations. Fourth-order tensor are denoted by barred letters, $\mathbb{C}$. The dot product between two second-order tensors $\mathbf{A}$ and $\mathbf{B}$ is denoted by $\mathbf{A}: \mathbf{B}=\sum_{i, j=1}^{N} A_{i j} B_{j i}$ where $A_{i j}$ and $B_{i j}$ are the tensor components. The average of a quantity over a region e.g. $D$ is denoted by $\langle\cdot\rangle_{D}$, while by $\langle\cdot \mid \cdot\rangle$, we denote the duality product.

\section{Computational design}

The considered micro-architectured materials are two-dimensional periodic assemblies of square unit cells. The unit cells are a two-phase composite with a strong and weak phase, e.g. polymer material and void, respectively. In the sequel, we will denote as shape the strong phase of the micro-architectured unit cell, e.g. polymer phase of the composite.

In this section, we present the micro-architectured material modelling using a level-set representation [32] in the unit cell, the computation of effective elastic tensors of the periodic micro-structure based on rigourous 

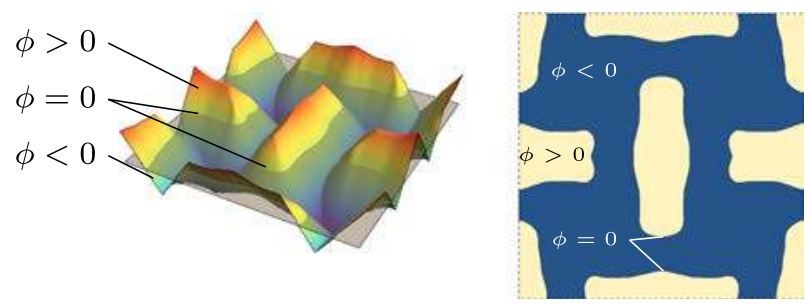

Fig. 1 Representation of the shape in the unit cell: a 3D representation of the level set sliced by the plane $\phi=0$ (left) projection of the level set on the Cartesian plane (centre), characteristic sets defined by the level set, i.e. void and material phases and their reciprocal boundary (right)

mathematical theory $[1,8,28,35]$ and a shape optimization method [3,42] to reach a target homogenized elasticity tensor.

\subsection{Shape of a unit cell and homogenized elastic tensor}

We denote by $Y$ the rescaled unit cell, of coordinates $\mathbf{y} \in[-1 / 2,1 / 2]^{2}$. The strong phase of the microarchitectured unit cell, i.e. the shape, is denoted by $\omega$ and is represented by a real-valued auxiliary level-set function $\phi$. The principle of the level-set method is to implicitly define the interface of a shape via the zero level set of $\phi$ [see Eq. (2.1) and Fig. 1]. Following the ideas of section 2 in [2], the level-set function serves as a base to define the local stiffness tensor $\mathbb{C}(\mathbf{y})$ in $Y$ as a smooth interpolation between the strong phase and the weak phase, e.g. polymer and void material properties, respectively.

$$
\left\{\begin{array}{llrl}
\phi(\mathbf{y})=0 & \text { if } \mathbf{y} \in \partial \omega \cap Y, & \text { (boundary) } \\
\phi(\mathbf{y})>0 & \text { if } \mathbf{y} \in Y \backslash \omega, & \text { (void) } \\
\phi(\mathbf{y})<0 & \text { if } \mathbf{y} \in \omega, & \text { (material) }
\end{array}\right.
$$

The material under consideration occupies a two-dimensional domain $\Omega$, described by a set of coordinates $\mathbf{x} \in \mathbb{R}^{2}$ and is modelled as a linear elastic composite with periodic structure. We introduce the small parameter $\epsilon$ as the ratio of the period of the structure to the typical size of the domain $\Omega$ and let $\epsilon \rightarrow 0$ to obtain the homogenized problem. The displacement $\mathbf{u}^{\epsilon}(\mathbf{x})$ satisfies the following problem:

$$
\begin{aligned}
-\operatorname{div}\left(\mathbb{C}^{\epsilon}(\mathbf{x})\right) \mathbf{u}^{\epsilon}(\mathbf{x}) & =\mathbf{f}(\mathbf{x}) & \text { in } \Omega, \\
\mathbf{u}^{\epsilon}(\mathbf{x}) & =\mathbf{0} & \text { on } \partial \Omega .
\end{aligned}
$$

where $\mathbb{C}^{\epsilon}(\mathbf{x}) \equiv \mathbb{C}(\mathbf{x} / \epsilon)$ represent a fourth-order stiffness tensor which is positive definitive and $\mathbf{f}(\mathbf{x})$ is a known body force.

Assume that $\mathbf{u}^{\epsilon}$ has a two-scale expansion of the form:

$$
\mathbf{u}^{\epsilon}(\mathbf{x})=\sum_{\alpha=0}^{+\infty} \epsilon^{\alpha} \mathbf{u}_{\alpha}(\mathbf{x}, \mathbf{y}), \quad \mathbf{y}=\frac{\mathbf{x}}{\epsilon} .
$$

This leads to a series of problems for different orders of $\epsilon$ : at order $\epsilon^{-2}$, we obtain that $\mathbf{u}_{\mathbf{0}}(\mathbf{x}, \mathbf{y}) \equiv \mathbf{u}_{\mathbf{0}}(\mathbf{x})$. At order $\epsilon^{-1}$, we obtain the displacement field solutions of the unit cell problems. At order $\epsilon^{0}$, we obtain the linear elastic constitutive equation averaged over the unit cell, yielding the following explicit energy formulation of the homogenized elastic tensor $\mathbb{C}^{H}$, expressed in terms of its Cartesian components as:

$$
C_{i j k \ell}^{H}=\int_{Y} \mathbb{C}(\mathbf{y})\left(\mathbf{E}^{i j}+\boldsymbol{\varepsilon}\left(\chi^{i j}\right)\right):\left(\mathbf{E}^{k \ell}+\boldsymbol{\varepsilon}\left(\chi^{k \ell}\right)\right) \mathrm{d} \mathbf{y},
$$

where

- $\mathbf{E}^{k \ell}$ designates a constant strain over the unit cell, resulting from the zero-order displacement $\mathbf{u}_{0}$. In $2 \mathrm{D}$, there are three independent unit strain fields, namely the horizontal unit strain $\mathbf{E}^{11}=(1,0,0)^{T}$, the vertical strain $\mathbf{E}^{22}=(0,1,0)^{T}$ and the shear unit strain $\mathbf{E}^{12}=(0,0,1)^{T}$.

$-\chi^{k \ell}$ represent the displacement fields, solution of the following linear elastic problems with periodic boundary conditions: 
Find admissible displacement $\chi^{k l}$ such that

$$
\int_{Y} \mathbb{C}(\mathbf{y})\left(\mathbf{E}^{k \ell}+\boldsymbol{\varepsilon}\left(\chi^{k \ell}\right)\right): \boldsymbol{\varepsilon}(\mathbf{v}) \mathrm{d} \mathbf{y}=0
$$

where $\mathbf{v}$ are admissible displacement vectors, i.e. with zero mean value and adequate smoothness.

\subsection{Elastic stiffness with orthotropic symmetry}

The effective stiffness tensor $\mathbb{C}^{H}$ in (2.4) carries a natural orthotropic material symmetry, provided that $\mathbb{C}$ is isotropic [35]. The linear elastic constitutive equation averaged over the unit cell relating the mean stress and strain tensors, denoted as $\sigma^{H}$ and $\varepsilon^{H}$, respectively, has therefore the following expression for the twodimensional problems under consideration:

$$
\begin{aligned}
\boldsymbol{\sigma}^{H} & =\mathbb{C}^{H} \boldsymbol{\varepsilon}^{H} \\
\text { where: } \quad \boldsymbol{\sigma}^{H} & =\langle\boldsymbol{\sigma}\rangle_{Y}, \quad \boldsymbol{\varepsilon}^{H}=\langle\boldsymbol{\varepsilon}\rangle_{Y} .
\end{aligned}
$$

In 2D elasticity, the components of $\mathbb{C}^{H}$ in matrix notation and in Cartesian coordinates read:

$$
\left(\begin{array}{c}
\sigma_{11}^{H} \\
\sigma_{22}^{H} \\
\sigma_{12}^{H}
\end{array}\right)=\left(\begin{array}{ccc}
C_{1111}^{H} & C_{1122}^{H} & 0 \\
C_{1122}^{H} & C_{2222}^{H} & 0 \\
0 & 0 & C_{1212}^{H}
\end{array}\right)\left(\begin{array}{c}
\varepsilon_{11}^{H} \\
\varepsilon_{22}^{H} \\
2 \varepsilon_{12}^{H}
\end{array}\right)
$$

Alternatively, one could express the effective strain as a function of the effective stress with the following effective material tensor:

$$
\left(\begin{array}{c}
\varepsilon_{11}^{H} \\
\varepsilon_{22}^{H} \\
2 \varepsilon_{12}^{H}
\end{array}\right)=\left(\begin{array}{ccc}
1 / E_{1} & -v_{12} / E_{2} & 0 \\
-v_{21} / E_{1} & 1 / E_{2} & 0 \\
0 & 0 & 1 / G
\end{array}\right)\left(\begin{array}{c}
\sigma_{11}^{H} \\
\sigma_{22}^{H} \\
\sigma_{12}^{H}
\end{array}\right)
$$

where $E_{i}$ denote the homogenized Young moduli, $v_{i j}$ denote the Poisson's ratios and $G$ denotes the homogenized shear modulus. Let us further remark that by symmetry of the elastic compliance matrix, the following ratios have to be equal:

$$
\frac{\nu_{12}}{E_{2}}=\frac{\nu_{21}}{E_{1}}
$$

The elastic moduli, $C_{i j k l}^{H}$, can equally be expressed in terms of the compliance moduli, i.e. Young moduli and Poisson's ratios: $C_{1111}^{H}=\left(1-v_{12} v_{21}\right)^{-1} E_{1}, C_{2222}^{H}=\left(1-v_{12} v_{21}\right)^{-1} E_{2}, C_{1122}^{H}=v_{21}\left(1-v_{12} v_{21}\right)^{-1} E_{1}$, $C_{2211}^{H}=v_{12}\left(1-v_{12} v_{21}\right)^{-1} E_{2}$ with $C_{1122}^{H}=C_{2211}^{H}$ as can be easily obtained from the inversion of the corresponding matrices. A simple calculation immediately yields:

$$
\nu_{12}=\frac{C_{1122}^{H}}{C_{2222}^{H}} \quad \text { and } \quad v_{21}=\frac{C_{1122}^{H}}{C_{1111}^{H}} .
$$

Moreover, the homogenized Poisson's ratio $v_{i j}$ are equally denoted effective Poisson's ratio to highlight their reference to the homogenized unit cell. For example, $v_{12}$ characterizes the contraction of the structure in the direction of $O y$ axis when the cell stretched in the direction of $O x$ axis and in general $v_{12} \neq v_{21}$. However, if the micro-architecture of the unit cell obeys "cubic" symmetry, we have $C_{1111}^{H}=C_{2222}^{H}$, and we trivially obtain that $E_{1}=E_{2}=E^{*}$ and $v_{12}=v_{21}=v^{*}$. 


\subsection{Shape optimization of the micro-structures}

Next, we discuss the framework of the optimization problem without presenting the details of the algorithm which is beyond the scope of the paper. A detailed discussion is given by the authors in [31].

Assume that the unit cell $Y$ is the working domain and consider $\omega$ an open and bounded subset of $Y$ representing the shape of the architectured micro-structure. The optimization problem seeks to find the shape $\omega$ such that the effective stiffness of the material reaches a given target value $\mathbb{C}^{t}$. The problem can be formulated as constrained minimization problem. The distance between the effective elastic moduli over the unit cell and target elastic moduli can be measured by the following cost functional:

$$
\mathcal{J}(\omega)=\frac{1}{2}\left\|\mathbb{C}^{H}(\omega)-\mathbb{C}^{t}\right\|_{\eta}^{2}
$$

where $\|\cdot\|_{\eta}$ is the weighted Euclidean norm, $\mathbb{C}^{t}$ is the target elastic tensor, and $\eta$ are the weight coefficients. We define a set of admissible shapes contained in the working domain $Y$ and have a prescribed volume by $\mathcal{U}_{a d}=\left\{\omega \subset Y\right.$ such that $\left.|\omega|=V^{t}\right\}$. Hence, the optimal shape design of the micro-architecture can be formulated as the following optimization problem:

$$
\begin{gathered}
\inf _{\omega \subset \mathcal{U}_{a d}} \mathcal{J}(\omega), \\
\chi^{k l} \text { satisfies (2.5). }
\end{gathered}
$$

In practice, the volume constraint is enforced using a Lagrange multiplier (the technique for updating the Lagrange was based upon the works of Allaire and Pantz [4]).

\subsection{Numerical algorithm}

The optimization of $\mathcal{J}(\omega)$ is carried out by advecting an initial shape $\omega_{0}$ with velocity $v$ obtained from the shape derivative $\left\langle\mathcal{J}^{\prime}(\omega) \mid \boldsymbol{\theta}\right\rangle$ in the direction $\boldsymbol{\theta}$ (see Allaire et al. [3] or Wang et al. [42]). The advection is realized by solving the Hamilton-Jacobi equation,

$$
\phi_{, t}+\mathbf{v} \cdot|\nabla \phi|=0
$$

where $\mathbf{v}$ is the velocity of the interface computed from the shape derivative $\left\langle\mathcal{J}^{\prime}(\omega) \mid \boldsymbol{\theta}\right\rangle$.

The numerical algorithm can be summarized in the following steps:

(i) Initialize the level set $\phi_{0}$ corresponding to the initial shape $\omega_{0}$.

(ii) Update the level set $\phi_{0}$ using the signed distance function $d_{\omega_{0}}$.

(iii) Iterate until convergence for $k \geq 0$ :

a. Calculate the local solutions $\bar{\chi}_{k}^{m \ell}$ for $m, \ell=1,2$ by solving the linear elasticity problem in $Y$.

b. Deform the domain $\omega_{k}$ by solving the above Hamilton-Jacobi equation. The new shape $\omega_{k+1}$ is characterized by the level set $\phi_{k+1}$ after a time step $\Delta t_{k}$. The time step $\Delta t_{k}$ is chosen so that $\mathcal{J}\left(\omega_{k+1}\right) \leq \mathcal{J}\left(\omega_{k}\right)$.

(iv) If needed for stability reasons, re-initialize the level-set functions $\phi_{k}$.

The complete algorithm as well as several examples are presented in Nika and Constantinescu [31]. Additional mathematical results and algorithmic issues can be found in the works of Allaire, Jouve and Toader [3], Allaire et al. [2] and Wang et al. [42] for more details about the mathematical results and algorithmic issues for the solution method. Let us remark that the algorithm does not allow for nucleations of voids. However, the level-set method is well-known to handle easily topology changes, i.e. merging or cancellation of holes. Therefore, algorithm is able to perform topology optimization if the number of holes of the initial design is sufficiently large and converges smoothly to a (local) minimum which strongly depends on the initial topology. Next, for each numerical example, we provide the initials guesses for the shapes which are typically a plate filled with holes, as illustrated in Figs. 2a, 4a and 5a. 


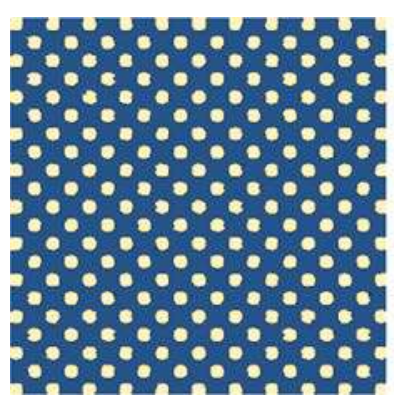

(a)

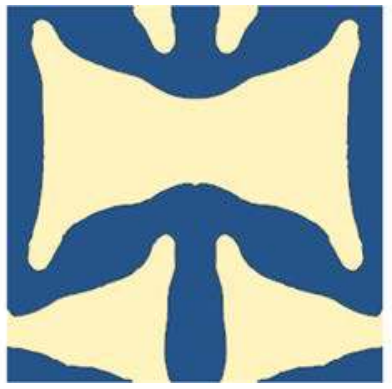

(b)

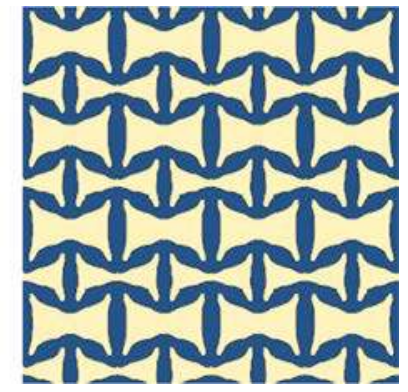

(c)
Material

Void

Fig. 2 Initial and final shape of the micro-structures: a initial shape consisting of a series of "circular" micro-perforations, $\mathbf{b}$ final, optimal, shape of the unit cell after 100 iterations, $\mathbf{c}$ final, optimal, shape of the periodic material

\subsection{Examples of obtained micro-structures}

In all the examples that follow, the Young's modulus was set to $E_{\mathrm{m}}=0.91 \mathrm{MPa}$ for the strong phase (material) and $E_{v}=0.91 \times 10^{-3} \mathrm{MPa}$ for the weak phase (void). The Poisson's ratio was set to $v=0.3$ for both phases. Under the plane stress assumption, the components of the elastic tensor of the base material are $C_{1111}^{m}=C_{2222}^{m}=1.0 \mathrm{MPa} ; C_{1122}^{m}=0.3 \mathrm{MPa} ; C_{1212}^{m}=0.35 \mathrm{MPa}$. The quadratic unit cell $Y$ was meshed with a structured symmetric grid of $100 \times 100$ quadrangular each formed of four equal triangular linear elements $(P 1)$. All computations were carried out using an in house programming of the preceding algorithm [31] operating on FreeFEM++ software [19]. The optimization is assumed to be terminated when 100 iterative steps are reached.

The main intention of the present work was to design micro-architectured materials exhibiting an effective negative Poisson's ratio. However, in all examples, the target objective was defined only in terms of the coefficients $C_{1111}^{H}, C_{1122}^{H}, C_{2222}^{H}$ using relation (2.10). The shear coefficient $C_{1212}^{H}$ as well as the $C_{1211}^{H}$ and $C_{1222}^{H}$ coefficients were left free. Therefore, only the elastic moduli of the unit cell corresponding to the direction 11 and 22 directions of strain and stresses were controlled.

Example 1 The first micro-structure to be optimized is a structure whose target effective Poisson's ratio is equal $v^{t}=-1.0$. The volume constraint was set to $V^{t}=50 \%$. We further note that for this structure, we enforced a symmetry of the shape along the vertical axis, by symmetrizing the level-set function after each iteration in the algorithm. The initial and final shapes of the micro-structure on the unit cell and as a periodic material are represented in Fig. 2.

The final shape can be characterized as an re-entrant honeycomb structure and looks similar to the designs imagined by Almgren [6]. Its homogenized coefficients, given in Table 1, show that the structure exhibits an effective orthotropic behaviour and a simple calculation yields $v_{12}=-1.25$ and $\nu_{21}=-0.42$. Hence, the expansion of the structure along the $O y$ axis when stretched in the $O x$ axis is larger than the expansion along the $O x$ axis when stretched in the $O y$ axis. This non-symmetric effect has been enabled as the symmetry relation was only imposed along the $O y$ axis in the algorithm.

The convergence history of the cost functional and of the volume constraint shown in Fig. 3 shows that the target coefficient got stabilized in slightly more than 20 iteration and that the later iteration contributed only to small improvements without bringing the cost functional to less than 0.06 which corresponds to $92 \%$ decrease in the initial value. The gap with respect to the target moduli is read from Table 1. It is interesting to remark that the final optimized micro-structure has a shear moduli close to 0 . However, the final effective Poisson's ratio is close to the set target as will be discussed in the comparison with the printed samples. The volume constraint has a different evolution than the cost functional with an initial increase given by the initial evolution of the holes and then a fast and a slow evolution which lies within the proposed range of the constraint.

Example 2 For the second micro-structure to be optimized, the target effective Poisson's ratio was also $v^{t}=$ -1 . The target tensor possesses a cubic symmetry, meaning the desired mechanical properties along the $O x$ and $O y$ axis should be equal. This time, the void volume fraction constraint was of an inequality type, and was set to $16 \% \leq V^{t} \leq 60 \%$. To counter the loss of symmetry observed in the previous example, a symmetry of the shape was enforced along both the $O x$ axis and $O y$ axis, by symmetrizing the level-set function during 
Table 1 From left column to right column: target elastic stiffness tensor $\mathbb{C}^{t}$, final elastic tensor $\mathbb{C}^{H}(\omega)$, target Poisson's ratio $v^{t}$, final effective Poisson's ratio $v^{*}$ unit cell shape $\omega$ for the discussed examples

\begin{tabular}{lllll}
\hline Example & $\mathbb{C}^{t}$ & $\mathbb{C}^{H}(\omega)$ & $v^{t}$ & $v^{*}$ \\
\hline 1 & $\left(\begin{array}{cccc}0.1 & -0.1 & 0 \\
-0.1 & 0.1 & 0 \\
0 & 0 & G\end{array}\right)$ & $\left(\begin{array}{ccc}0.12 & -0.05 & 0 \\
-0.05 & 0.04 & 0 \\
0 & 0 & 0.006\end{array}\right)$ & -1. & $\{-1.25,-0.42\}$ \\
2 & $\left(\begin{array}{cccc}0.1 & -0.1 & 0 \\
-0.1 & 0.1 & 0 \\
0 & 0 & G\end{array}\right)$ & $\left(\begin{array}{ccc}0.12 & -0.05 & 0 \\
-0.05 & 0.12 & 0 \\
0 & 0 & 0.003\end{array}\right)$ & -1. & -0.42 \\
3 & $\left(\begin{array}{cccc}0.2 & -0.1 & 0 \\
-0.1 & 0.2 & 0 \\
0 & 0 & G\end{array}\right)$ & $\left(\begin{array}{ccc}0.19 & -0.09 & 0 \\
-0.09 & 0.19 & 0 \\
0 & 0 & 0.6\end{array}\right)$ & -0.47 \\
\hline
\end{tabular}

Let us remark that the bottom two structures on the right column carry cubic symmetry, while the top structure on the right column carries orthotropic symmetry

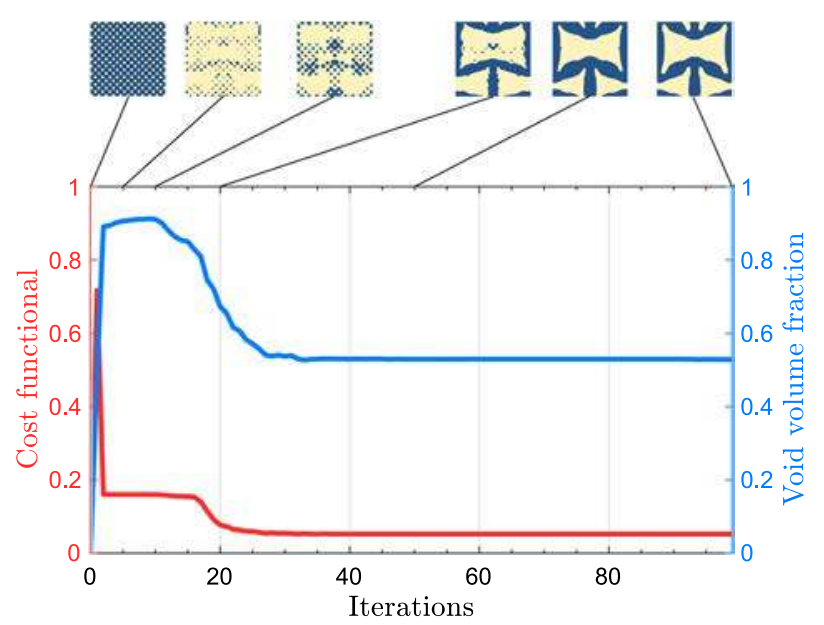

Fig. 3 Evolution of the cost functional (in red) and volume constraint V (in blue) during 100 iterations (colour figure online)

the algorithmic iterations. The initial and final shapes of the micro-structure on the unit cell and as a periodic material are shown in Fig. 4, while the target and final elastic moduli are shown in Table 1.

As prescribed, the resulting structure exhibits a "cubic" symmetry. The computed effective Poisson's ratio is $v^{*}=v_{12}=v_{21}=-0.42$. By comparing at the target and the obtained elastic tensor, one can remark that the diagonal elastic moduli $C_{1111}^{H}$ and $C_{2222}^{H}$ are fairly close to the target but the shear $C_{1122}^{H}$ fails at attaining the desired properties of $v^{t}=-1$. This suggests that there is a trade-off between symmetrical tensor and extreme negative Poisson's ratio in the optimization algorithm. This will further be discussed in the following section. Finally, let us remark that the final volume ratio is at approximately $36 \%$ and lies in the middle of the imposed interval. As before, the final optimized micro-structure has a shear modulus $C_{1212}^{H}$ close to 0 .

Example 3 For the third micro-structure to be optimized, the target effective Poisson's ratio was $v^{t}=-0.5$. The target tensor possesses a cubic symmetry, meaning the desired mechanical properties along the $O x$ and $O y$ axes should be equal. The void volume fraction constraint is set to $V^{t}=40 \%$ and is updated the same way as in the first example. As in Example 2, a symmetry of the shape was enforced along the $O x$ and $O y$ axes.

The final shape is of the rotating unit type, discussed in Grima et al. [18]. As prescribed, the resulting structure exhibits a "cubic" symmetry. The computed effective Poisson's ratio is $v^{*}=v_{12}=v_{21}=-0.47$. By comparing the target and the obtained elastic tensor, one can remark that the final elastic moduli are fairly 

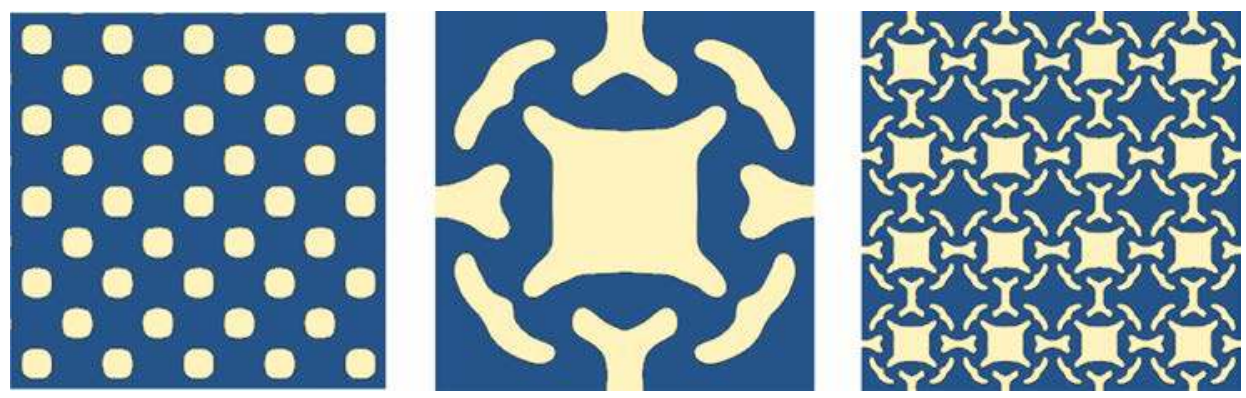

Material

Void

Fig. 4 The design process of the material. Image a depicts the initial guess of the micro-structure, $\mathbf{b}$ depicts the optimal microstructure after 100 iterations, $\mathbf{c}$ depicts the macroscopic material assembled periodically from the optimal unit cell of image $\mathbf{b}$

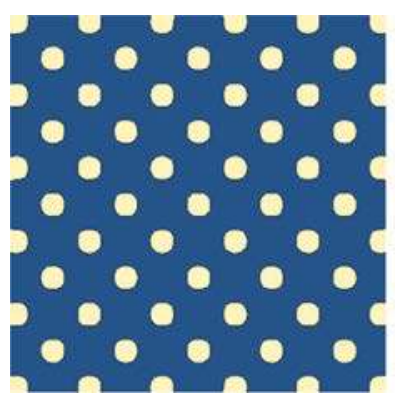

(a)

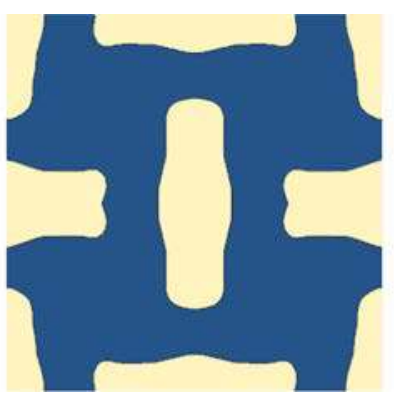

(b)

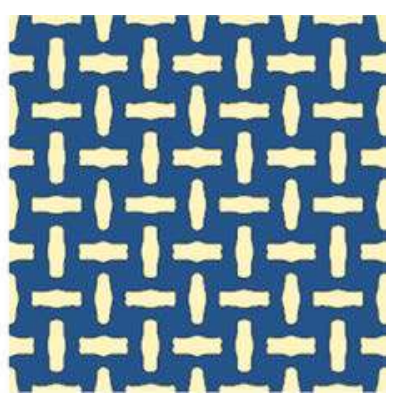

(c)
Material

Void

Fig. 5 The design process of the material. Image a depicts the initial guess of the micro-structure, $\mathbf{b}$ depicts the optimal microstructure after 100 iterations, $\mathbf{c}$ depicts the macroscopic material assembled periodically from the optimal unit cell of image $\mathbf{b}$

close to the target and that this structure has a shear modulus $C_{1212}^{H}$ which is of the same order of magnitude as the other moduli. Finally, let us remark that the final volume ratio is at approximately $43 \%$ and lies in the vicinity of the imposed value (Fig. 5).

\subsection{Representation of the examples in the space of elastic stiffness}

The elastic tensor, $\mathbb{C}^{H}$, governs the overall effective material response to an applied load. Since we assumed that the material tensor characterizing the micro-structure is positive definite then $\mathbb{C}^{H}$ is also positive definite. In terms of the physical parameters $E_{1}, E_{2}, v_{12}, v_{21}$, the positive definiteness of the effective material tensor requires that the stiffness tensor is positioned with the following stability bounds (see the works of Ting and Chen [39] for a detailed discussion):

$$
\left|\nu_{12}\right| \leq \sqrt{\frac{E_{1}}{E_{2}}}, \quad\left|\nu_{21}\right| \leq \sqrt{\frac{E_{2}}{E_{1}}} .
$$

In Fig. 6, we plot the Poisson's ratios against the stability bounds of certain optimal shapes from some recent articles published and see how they compare with our own optimized micro-structures.

A material that would have $E_{1}=E_{2}$ and $v_{21}=v_{12}=v^{*}=-1$ would fall on the lower stability bound. From the graph of Fig. 6, we understand that the structure has to loose its "cubic" symmetry and accept an important stiffness unbalance between the directions 1 and 2, expressed by the ratio $E_{2} / E_{1}$ to reach one extreme negative Poisson's ratio.

The shapes from the literature that have been designed by the optimization of a level-set function, share generic features: lattice architectures with re-entrant corners (like re-entrant honeycomb) or rotating semi-rigid units connected by flexible hinges. Moreover, the orthotropic structures with $E_{2} / E_{1}<0.8$ are only lattice architectures with re-entrant corners. Let us further mention the recent contributions of [44] who designed a 


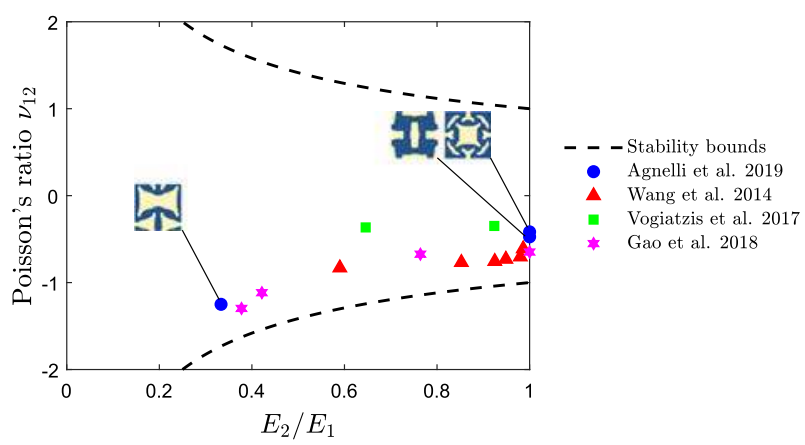

Fig. 6 Optimal shapes obtained from this article as well as from the literature $[16,40,43]$ plotted against the stability bounds. Remark that extreme Poisson's ratio (i.e. below -1 ) are reachable for anisotropic structures

chiral bi-material micro-architecture with $E_{2} / E_{1} \approx 0.65$ and an effective Poisson's ratio $v_{12}=-0.8$ using the SIMP-optimization method, which would also fill in the data cloud of Fig. 6. Lastly, the examples from literature, aimed to reach a maximal negative Poisson's ratio, fall at a certain distance from the inferior stability bound. We therefore understand the need for tighter bounds to be used as a guide to effectively explore the ability of the algorithm to ascertain which elastic moduli are attainable in future designs.

\section{Analysis of fabricated polymer structures}

\subsection{Manufacturing process: equipment and materials}

The optimal shapes have been additively manufactured with digital light processing stereolithography technology (DLP) using a commercial Ember 3D printer. A digital projector screen flashes a single image of each layer across the entire surface of the vat filled of photosensitive liquid resin at once, causing chains of molecules to link and thus forming solid polymer. The process is repeated until the 3D model is complete. Then, the vat is drained of liquid, revealing the solidified model and the solid model is washed with a solvent.

The printer has a resolution of $50 \mu \mathrm{m}$, corresponding to 1 pixel in the digital projector screen, and a range of the processing layer thickness of $10-100 \mu \mathrm{m}$. The largest processing build volume is $64 \mathrm{~mm} \times 40 \mathrm{~mm} \times$ $134 \mathrm{~mm}$ (note that $64 \mathrm{~mm} \times 40 \mathrm{~mm}$ correspond to a $1280 \times 800$ pixels picture). For a thickness of $25 \mu \mathrm{m}$ per layer, the speed range is of $18 \mathrm{~mm} / \mathrm{h}$. The printable minimal feature size of the specimens is announced at $0.4 \mathrm{~mm}$ corresponding roughly to 8 pixels.

We selected a rubber-like material, commercially denoted as GM08b, ${ }^{1}$ as the base material because of its compliant nature. Figure 7 displays a representative tensile stress-strain curve of this material. As expected for a rubber-like material, it does not display an ideal linear elastic behaviour; it exhibits a gradual variation of the stiffness with increasing strain.

The optimal shapes obtained in Example 1-3 (see Figs. 2, 4,5) are represented by the final level-set function. The later presents a smooth variation between values corresponding to the two materials in a neighbourhood of their interface, and therefore the level-set representation has been binarized and extruded in the $O z$ direction in order to create a 3D object. More precisely, the 3D-printed samples have been produced by the following procedure:

(i) Binarize the level-set function representing by shape optimization.

(ii) Create a periodic array for each sample: $8 \times 6$ unit cells for example 1, $5 \times 4$ unit cells for Examples 2 and 3. The final result was a binarized $1280 \times 800$ pixels image (see Fig. 8 for details).

(iii) Extrude the preceding image to obtain the 3D sheet of the desired height. The final dimensions of the printed samples are $64 \times 38 \times 6 \mathrm{~mm}$ for Example 1 and $64 \times 40 \times 6 \mathrm{~mm}$ for Examples 2 and 3 .

(iv) Print the files with the following processing parameters: laser power was $5 \mathrm{~W}$, the exposure time $1 \mathrm{~s}$ per layer and the layer thickness was $50 \mu \mathrm{m}$.

(v) Wash the samples in an isopropanol bath for $5 \mathrm{~min}$.

(vi) Post-cure the samples for $30 \mathrm{~min}$ in an UV oven at $2000 \mathrm{~W}$.

\footnotetext{
${ }^{1}$ Characteristics of this material can be found in the manufacturers data sheet (see https://dl.airtable.com).
} 


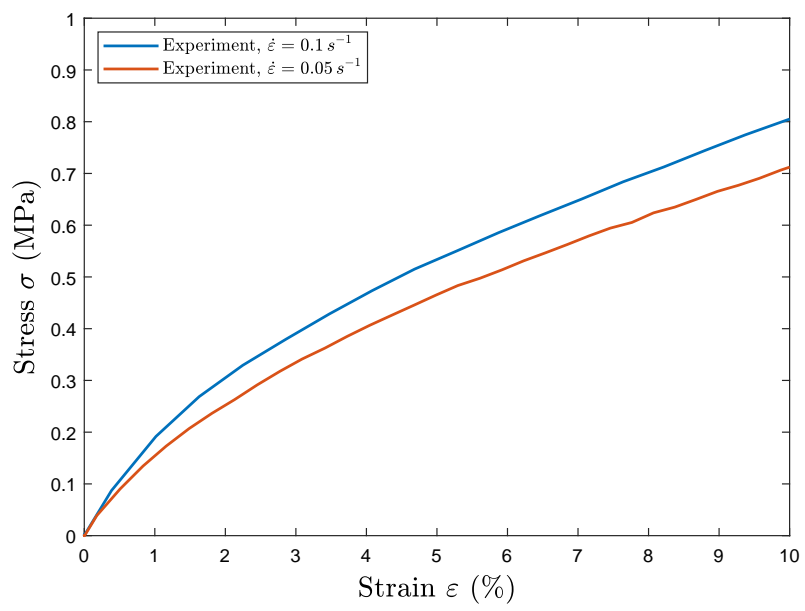

Fig. 7 Base material response to uniaxial tensile loading. Homogeneous stress-strain curves

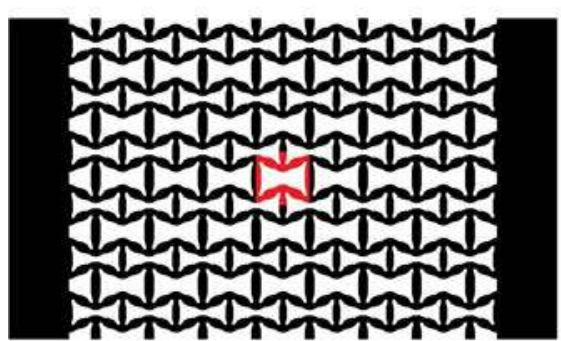

Example 1: Input design

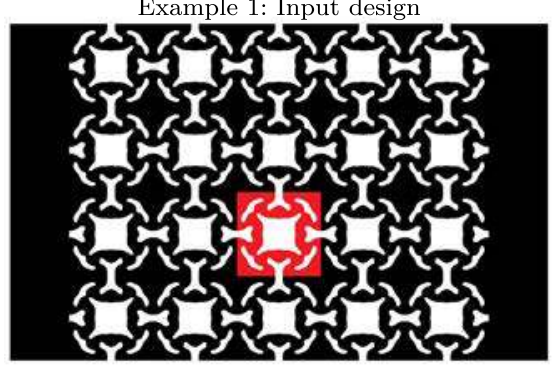

Example 2: Input design

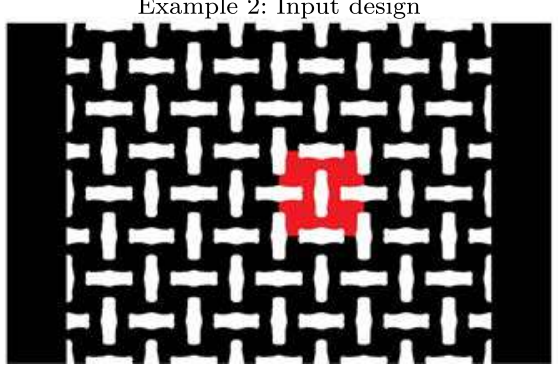

Example 3: Input design

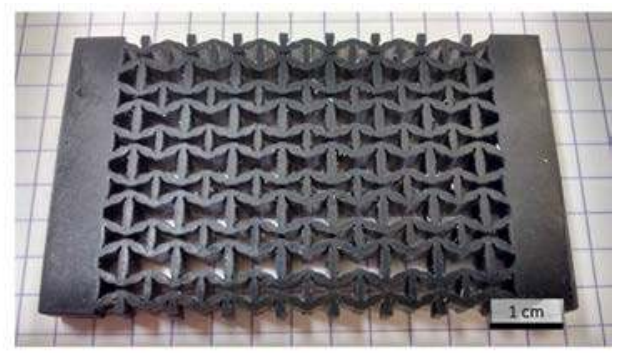

Example 1: Fabricated result

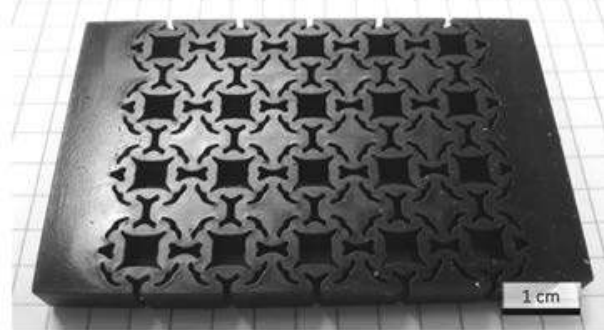

Example 2: Fabricated result

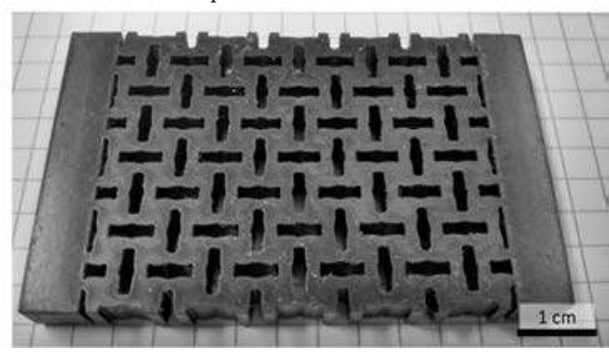

Example 3: Fabricated result

Fig. 8 Fabricated specimen from the examples of Sect. 2. Digital image fed into the 3D printer (left) and final printed specimen (right). The red coloured unit cells were the cells observed during the digital image correlation measurements (colour figure online)

3.2 Testing and full-field displacement measurement using digital image correlation (DIC)

A series of uniaxial static tensile tests were undertaken to assess the tensile properties of the auxetic lattice structures by using a home-made testing machine with a symmetric displacement of the two cross-heads and 


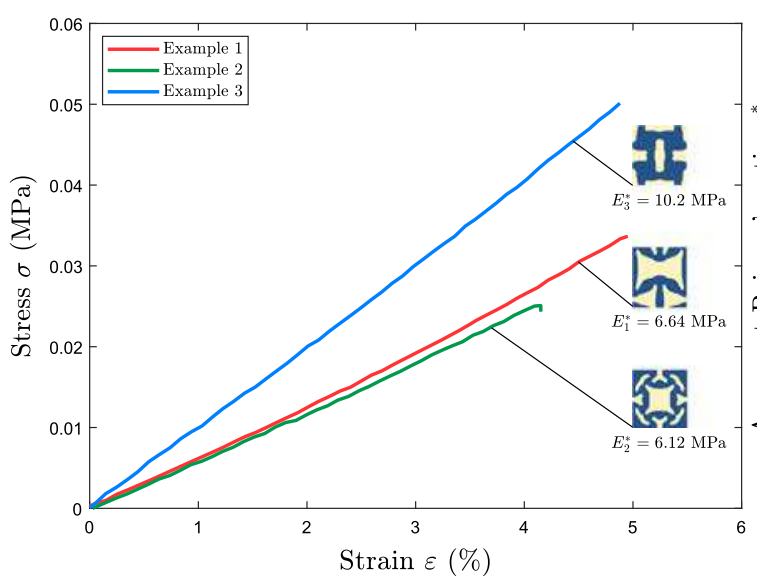

(a)

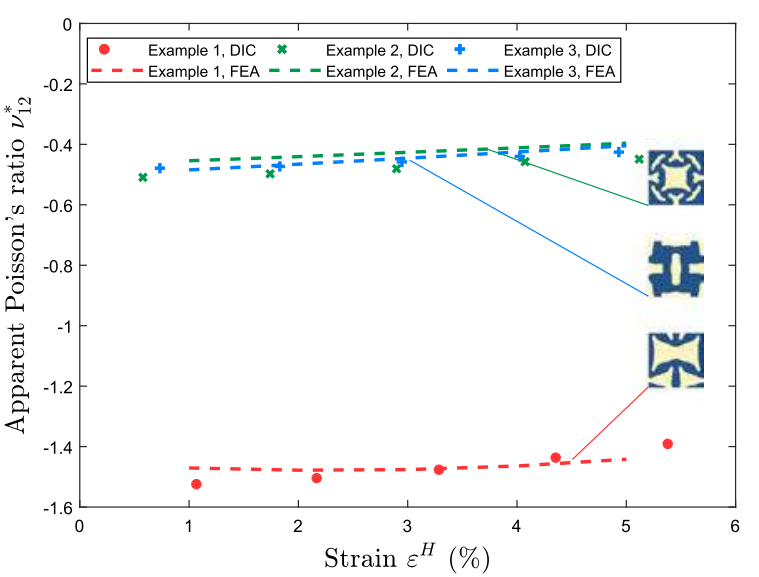

(b)

Fig. 9 Image a contains plots of the effective stress-strain curves for all three examples overlaid in the same plot obtained experimentally by performing a uniaxial tensile test. One can clearly observe all three structures exhibit linear behaviour for strains up to 5\%. Image b shows a comparison of the evolution of the Poisson's ratio plotted as a function of the effective strain from measurements taken by digital image correlation versus computations made by finite element analysis. We can clearly observe a trend on all three materials of the loss of their auxeticity as the uniaxial strain increases beyond the 5\% mark

equipped with a $100 \mathrm{~N}$ load cell. The tensile tests were performed at a rate of $0.05 \mathrm{~mm} / \mathrm{s}$ up to $3 \mathrm{~mm}$, which correspond to a strain rate of $\dot{\varepsilon}=10^{-3} \mathrm{~s}^{-1}$ up to a maximal strain of $\varepsilon=5 \%$.

The tensile tests were recorded and used for full-field measurements by digital image correlation (DIC). The recordings were obtained using a high-resolution digital camera (Schneider Optics 8-bit camera with a Makro-UNIFOC 100/77 lens) mounted on the tensile testing machine, and grey-scale pictures with resolution of $4904 \times 3280$ were recorded every second during the loading. The camera was mounted on a perpendicular axes with respect to the plane of the specimen, which enables the direct use of a 2D DIC. To improve the precision of the measurement, a white speckle pattern was placed on the sample by airbrushing.

The DIC was performed using the CorrelManuV 2D (CMV) software, developed by Bornert [5]. The processed displacement field corresponds to a single unit cell in the middle of the structure at five different loading time steps, using a $100 \times 100$ grid, i.e. having 10,000 measurement points. For each node, the subset size was set to $20 \times 20$ pixels, while the searching area was set to $100 \times 100$ pixels. The measurement included a computation without transformation, i.e. rotation of the subset window and a re-optimization allowing transformations with a reduced searching area of $30 \times 30$ pixels.

\subsection{Experimental results}

The stress-strain response under a uniaxial tensile test along $e_{1}$ for the three materials is shown in Fig. 9a. One can easily observe a linear behaviour of the samples that up to a maximal strain of $5 \%$ strain despite the nonlinearity of the rubber-like base material in the same strain range. This indicates that the samples have an expected structural deformation where different parts of the "lattice" behave as rigid struts and deformable hinges. This effect will be highlighted by the DIC measurements discussed later.

One can directly observe a lateral expansion during the tensile extension indicating a negative Poisson's ratio for all the samples. The precise measurements of the Poisson's ratio corresponding to a single central unit cell are presented in Fig. 9b. The precise method for the computation of the Poisson's ratio of a single unit cell from DIC measurements was based on periodic homogenization assumptions and the details are presented in A. The results show that the initial effective Poisson's ratio was for all samples close to the announced values in the optimization process and was not degraded during the manufacturing process. During tensile loading, the effective Poisson's ratio tends to increase, indicating a decrease in the "auxeticity" of the samples of up to increases by $10 \%$ for a $5 \%$ strain.

Finite element computation was undertaken under the assumption of small strains, large displacements and plane stress using the finite element solver Cast 3M 2018 (http://www-cast3m.cea.fr). The mesh was obtained using image processing from the binarized images of the optimal level-set function and completed to 
Table 2 Mean strain components of the base material measured by DIC and computed by FEA on the unit cells from Fig. 10 for the structures in Examples 1-3, denoted by the superscript

\begin{tabular}{lcccccc}
\hline $\boldsymbol{\varepsilon}_{h}^{H}$ & $\mathrm{DIC}^{1} \%$ & $\mathrm{FEM}^{1} \%$ & $\mathrm{DIC}^{2} \%$ & $\mathrm{FEM}^{2} \%$ & $\mathrm{DIC}^{3} \%$ & $\mathrm{FEM}^{3} \%$ \\
\hline$\left\langle\varepsilon_{11}\right\rangle_{h}$ & 1.25 & 0.543 & 0.526 & 0.404 & 0.937 & 0.828 \\
$\left\langle\varepsilon_{11}\right\rangle_{h}$ & -1.01 & -0.284 & -0.450 & -0.204 & -0.643 & -0.407 \\
$\left\langle\varepsilon_{12}\right\rangle_{h}$ & 0.039 & 0.004 & 0.053 & 0.002 & 0.010 & 0.001 \\
\hline
\end{tabular}

The measurement corresponds to the maximal loading with an effective strain of $5 \%$

the sample geometry. The elastic material behaviour was defined as the tangent behaviour at the origin of the tensile curve of the material. The sample was loaded with a given resultant force at the clamps of the tensile machine.

Let us first remark that the evolution is close to predictions of the deformation of the samples obtained by the finite element method under the assumption of large displacements. Second, one can remark that the evolution of the Poisson's ratio with applied strain has already been observed and discussed in [12] on polymeric filament structure. Moreover, they arrived to correct the phenomenon up to $20 \%$ strain using a nonlinear material behaviour in the optimization process, see $[12,43]$ for more details on the subject. In the case of the optimization procedure presented here, the extension to nonlinear material behaviour is currently under investigation and will appear in subsequent work of the authors.

The displacements fields obtained using DIC permit a further comparison with predictions and give an insight of the deformation mechanism of the samples, i.e. how the structure moves and deforms. Figure 10 displays the measured and the computed vertical displacement, i.e. the $u_{y}$ displacement component of the central unit cell. A comparison of the values and the shapes of the colour maps exhibits a good match between the measurements and finite element prediction. Moreover the displacement fields permit to better understand the local movements of the micro-structure which conducts to the global auxeticity effect by combining almost rigid regions submitted to translations and rotations with local concentrated deformation exhibiting local hinges. A further comparison in terms of mean displacements over a unit cell between DIC measurements and FEM computations at the maximal loading of 5\% strain is given in Table 2 . The mean was computed only over the base material of a unit cell and does represent the mean deformation of the later. Nevertheless, this mean value represents the mean loading of the base material and one can notice that the micro-structure does not leave the region of $1 \%$ strain of the uniaxial tensile response of the base material shown in Fig. 7. In Fig. 10, one can equally notice the excellent quality of the printing process as the edges of the printed shapes observed on left column of Fig. 10 is close to the edges of the shape represented by the level set and displayed here as the border of the finite element meshes on the right column of Fig. 10.

By looking at the strain field of a unit cell (see Fig. 11), computed by finite elements from the displacement field in both full-field measurement and simulation, we can notice that the strain field is mostly concentrated on the hinges of the structure. This further emphasizes the predominance of structural deformation as a lattice structure with rods and hinges at small strain. A subtle effect of this prevalence is the small effect of the out of plane strain, which should otherwise be perceived as a difference between the 2D modelling during the optimization process and the complete 3D character of the polymer sheets. Let us also recall that the polymer is practically incompressible and exhibit therefore an important variation of thickness under tensile loading.

The next steps in the analysis of the micro-architectured material are the complete experimental measurement of its elastic tensor. Let us recall that the effective constitutive law (2.6) or alternatively (2.7) is a linear relation between the components of the effective stress and strain, from which the elastic moduli could be identified by a least square fitting. The main difficulty is that only the effective strain, $\boldsymbol{\varepsilon}^{H}$, can be directly measured from the experiment, see values in Table 3. However, as suggested in [34], the effective stress, $\boldsymbol{\sigma}^{H}$, can be numerically computed from the experimental applied forces if the geometry and the constitutive behaviour of the base material. As a consequence, $\mathbb{C}^{H}$, the effective elastic tensor of the design phase is obtained as a linear fit from $\varepsilon^{H}$ and $\sigma^{H}$. The computation can be performed on several unit cells of the specimen. In order to compare the values of the elasticity tensor $\mathbb{C}^{H}$ computed in the design phase, we have non-dimensionalized the resultant forces.

Specimens in Examples 2 and 3 have a cubic material symmetry, which leads to a system of three equations with three unknowns for each unit cell. One has to identify three moduli $C_{1111}=C_{2222}, C_{1122}, C_{1212}$ using the 11, 22, 12 strain and stress components. The estimated elastic moduli on the central unit cell, i.e. with position 

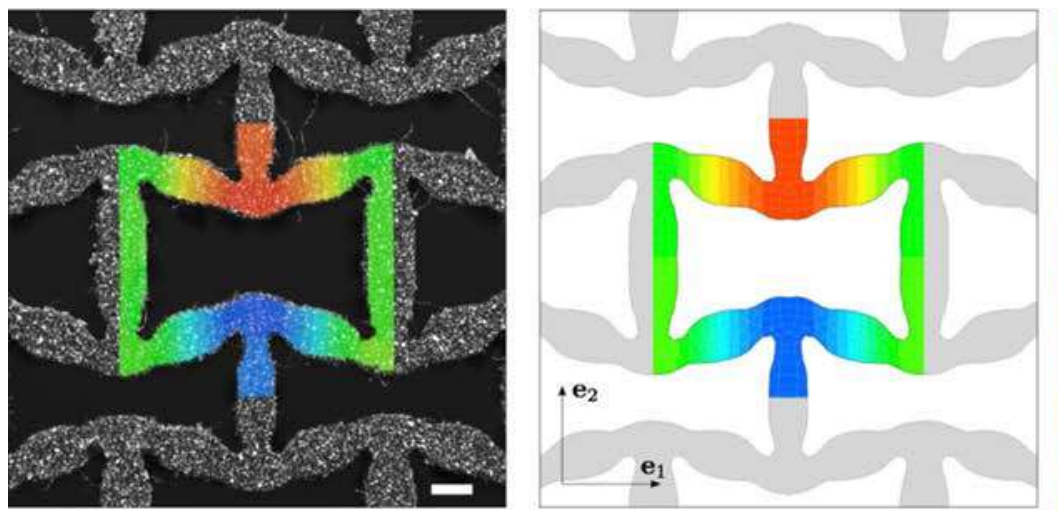

0.05

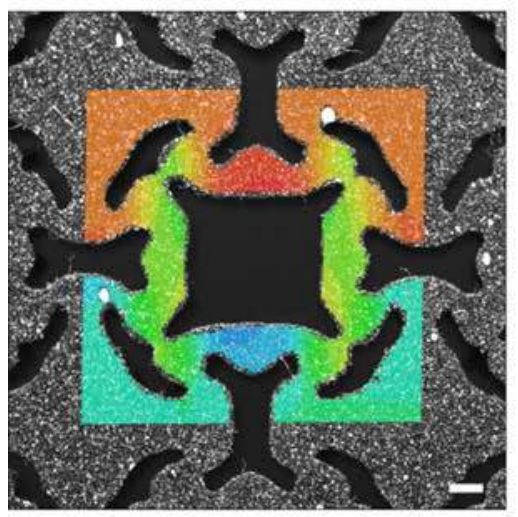

(a)

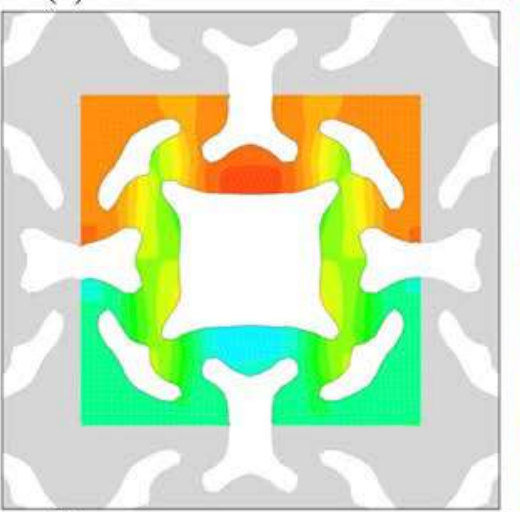

0.

(b)
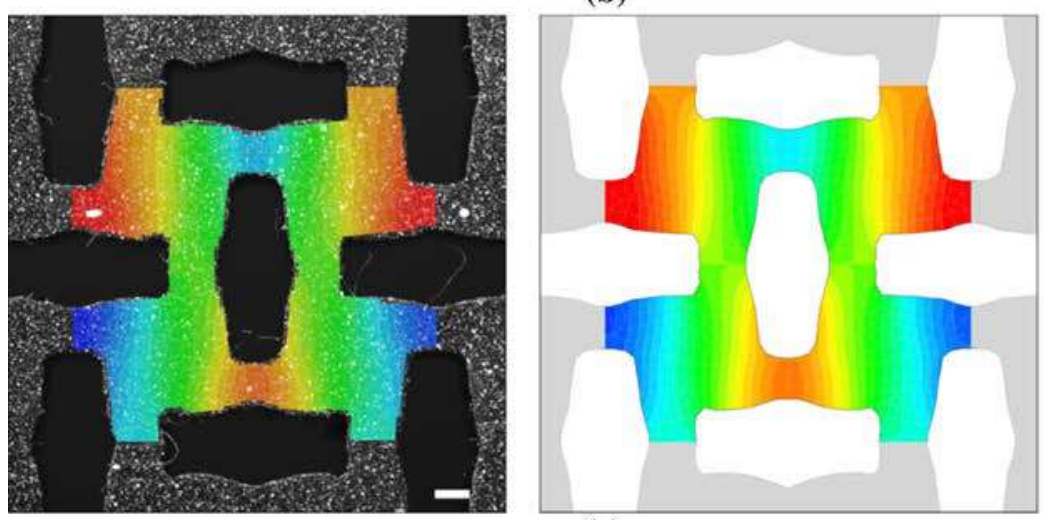

$-0.04$

0.01

0.

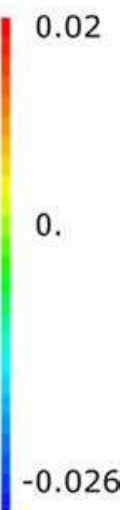

(c)

Fig. 10 Plots of the dimensionless values of the transverse displacement field along $e_{2}$ for each unit cell of the optimized structure. The samples are loaded along $e_{1}$ at $5 \%$ effective strain. The displacement field for the images on the left were measured using digital image correlation, while for the images on the right using finite element analysis. Image a is the optimized structure of Example 1, image b the optimized structure of Example 2, image $\mathbf{c}$ is the optimized structure of Example 3. Scale bar in all images is $1 \mathrm{~mm}$

$(3,3)$ and coloured red in Fig. 8 , are given in Table 3. The $C_{1212}$ moduli are missing as the signal-to-noise ratio of the effective shear strains and stresses was to small to provide meaningful value.

\section{Conclusion}

In this work, we used topology optimization methods to design optimal shapes that achieve a negative Poisson's ratio. By removing certain material constraints, e.g. isotropy, from the algorithm, we expanded the space of admissible shapes, and as a result, the algorithm was able to attain shapes with a Poisson's ratio below -1 . 

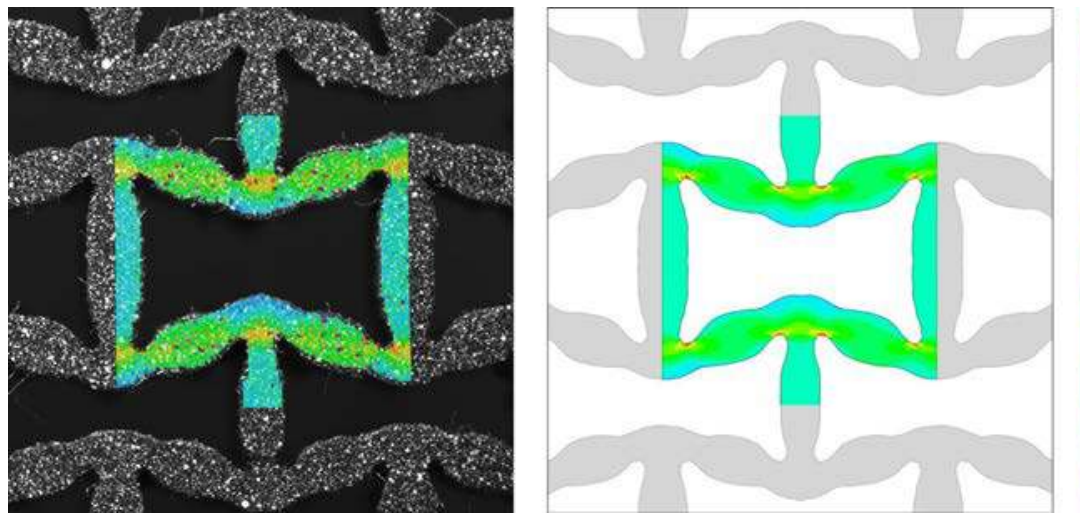

0 .

Fig. 11 Plot of the dimensionless values of the horizontal tensile strain field in a single unit cell of the optimized structure. The strain field for the image on the left was computed from the raw displacement field obtained by Digital Image Correlation, while for the image on the right using finite element analysis. We remark that our DIC approach generated some outliers that shall not be taken into account

Table 3 Comparison between the effective $\mathbb{C}^{H}(\omega)$ (see also 1) and measured elasticity tensor $\mathbb{C}^{H, \exp }(\omega)$ displayed in the left and centre column, respectively

\begin{tabular}{lllll}
\hline Example & $\mathbb{C}^{H}(\omega)$ & $\mathbb{C}^{H, \exp (\omega)}$ & $v^{*}$ & $v^{*, \exp }$ \\
\hline 2 & $\left(\begin{array}{cccc}0.12 & -0.05 & 0 \\
-0.05 & 0.12 & 0 \\
0 & 0 & G\end{array}\right)$ & $\left(\begin{array}{ccc}0.10 & -0.044 & 0 \\
-0.044 & 0.10 & 0 \\
0 & 0 & G\end{array}\right)$ & -0.42 & -0.44 \\
3 & $\left(\begin{array}{cccc}0.19 & -0.09 & 0 \\
-0.09 & 0.19 & 0 \\
0 & 0 & G\end{array}\right)$ & $\left(\begin{array}{ccc}0.204 & -0.09 & 0 \\
-0.09 & 0.204 & 0 \\
0 & 0 & G\end{array}\right)$ & -0.47 & -0.44
\end{tabular}

The right column displays the optimal shape in each case. We recall that the measured elasticity tensor $\mathbb{C}^{H, \exp }(\omega)$ was determined by combining DIC measurements and FEM computations

The effective elasticity tensor characterizing the material with Poisson's ratio below -1 is orthotropic, and although the theoretical problem of reachable elasticity tensors has been solved in the seminal work of Milton and Cherkaev [30], the algorithm suggests that the more we expand the space of admissible shapes by allowing shapes to deviate from isotropic symmetry the closer to the stability bounds the effective material approaches (see Fig. 6).

The results showed that optimal shapes could be directly printed without additional enhancement of the surface, which is a direct consequence of the smoothed interface technique used in the optimization. Moreover, the manufactured materials had the designed mechanical behaviour. The targeted elastic moduli and the underlying Poisson's ratios have been experimentally attained and the local material behaviour was close to predictions.

The local displacement field computed and measured on the micro-structure showed that measurements match numerical predictions. Moreover, one can observe from the strain field that the global deformation is composed of rigid regions an localized hinges, indicating that the structures behaves as rotating rigid units.

Acknowledgements This work is financed by the french-swiss ANR-SNF project MechNanoTruss (ANR-15-CE29-0024-01). The authors would like to express their gratitude to Chiara Daraio for fruitful discussion on the design of lattice structures and to Gregoire Allaire and Georgios Michailidis for lending their expertise on the numerical and algorithmic issues of the optimization. 


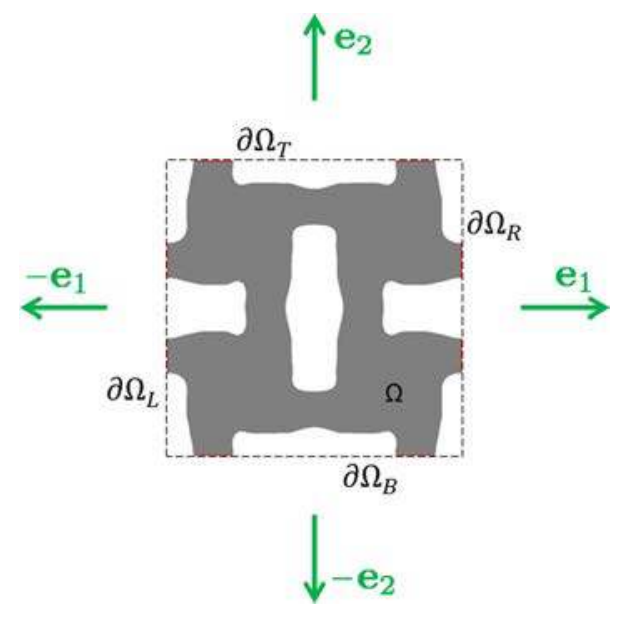

Fig. 12 Representation of a unit cell

\section{A computation of the effective Poisson's ratio}

This appendix reviews the mathematical approach that was used to measure/compute the effective Poisson's ratio of a unit cell in both measurements by digital image correlation and numerical estimation using a finite element method. For the following computation, we place ourselves in the case of small strain assumption (Fig. 12).

The effective material is supposed to carry a natural orthotropic material behaviour. The effective Poisson's ratio $v_{12}$, characterizing the transverse strain of the structure in the direction $\left(O, \mathbf{e}_{2}\right)$ axis when stretched in the direction $\left(O, \mathbf{e}_{1}\right)$, is defined as:

$$
v_{12}^{*}=\frac{C_{1122}^{H}}{C_{2222}^{H}}
$$

We remind that $C_{1122}^{H}$ and $C_{2222}^{H}$ are coefficients of the effective elastic stiffness tensor. In general $v_{12} \neq v_{21}$. During a uniaxial tensile test in the direction $\left(O, \mathbf{e}_{1}\right)$, Eq. (A.1) yields to the negative of the ratio of macroscopic transverse strain to macroscopic axial strain:

$$
v_{12}^{*}=-\frac{\varepsilon_{22}^{H}}{\varepsilon_{11}^{H}}
$$

In the small strain assumption, the strain field can be linearized as:

$$
\boldsymbol{\varepsilon}^{\mathbf{H}}=\langle\boldsymbol{\varepsilon}\rangle_{\Omega}=\frac{1}{2}\left(\langle\mathbb{F}\rangle_{\Omega}^{T}+\langle\mathbb{F}\rangle_{\Omega}\right)-\mathbb{I}
$$

where $\mathbb{F}$ is the average transformation gradient. Considering the small strain assumption:

$$
\langle\mathbb{F}\rangle_{\Omega}=\frac{1}{V_{\Omega}} \int_{\Omega}(\mathbb{I}+\nabla \mathbf{u}) \mathrm{d} \Omega
$$

Using Ostrogradsky's theorem, we can express the transformation gradient at the boundary $\partial \Omega$ :

$$
\langle\mathbb{F}\rangle_{\Omega}=\frac{1}{V_{\Omega}}\left(\int_{\Omega} \mathbb{I} d \Omega+\oint_{\Gamma} \mathbf{u} \otimes \mathbf{n d} \Gamma\right)
$$

Study of a unit cell

$$
\begin{aligned}
& \langle\mathbb{F}\rangle_{\Omega}=\mathbb{I}+\frac{1}{V_{\Omega}}\left(\int_{\partial \Omega_{T}} \mathbf{u} \otimes \mathbf{e}_{2} \mathrm{~d} \Gamma+\int_{\partial \Omega_{B}} \mathbf{u} \otimes\left(-\mathbf{e}_{2}\right) \mathrm{d} \Gamma+\int_{\partial \Omega_{R}} \mathbf{u} \otimes \mathbf{e}_{\mathbf{1}} \mathrm{d} \Gamma+\int_{\partial \Omega_{L}} \mathbf{u} \otimes\left(-\mathbf{e}_{\mathbf{1}}\right) \mathrm{d} \Gamma\right) \\
& \langle\mathbb{F}\rangle_{\Omega}=\mathbb{I}+\frac{1}{V_{\Omega}}\left[\begin{array}{l}
\int_{\partial \Omega_{R}} u_{1} d \Gamma-\int_{\partial \Omega_{L}} u_{1} \mathrm{~d} \Gamma \int_{\partial \Omega_{T}} u_{1} \mathrm{~d} \Gamma-\int_{\partial \Omega_{B}} u_{1} \mathrm{~d} \Gamma \\
\int_{\partial \Omega_{R}} u_{2} \mathrm{~d} \Gamma-\int_{\partial \Omega_{L}} u_{2} \mathrm{~d} \Gamma \int_{\partial \Omega_{T}} u_{2} \mathrm{~d} \Gamma-\int_{\partial \Omega_{B}} u_{2} \mathrm{~d} \Gamma
\end{array}\right]
\end{aligned}
$$


Thus, from Eq. (A.3):

$$
\left\{\begin{array}{l}
\varepsilon_{11}=\frac{1}{V_{\Omega}}\left(\int_{\partial \Omega_{R}} u_{1} \mathrm{~d} \Gamma-\int_{\partial \Omega_{L}} u_{1} \mathrm{~d} \Gamma\right) \\
\varepsilon_{22}=\frac{1}{V_{\Omega}}\left(\int_{\partial \Omega_{T}} u_{2} \mathrm{~d} \Gamma-\int_{\partial \Omega_{B}} u_{2} \mathrm{~d} \Gamma\right)
\end{array}\right.
$$

For each edge of the square unit cell, the integral of the contour is computed by integrating the displacement of the material in contact with the edge. In other words, the void phase is not considered in the computation.

$$
v_{12}^{*}=-\frac{\int_{\partial \Omega_{T}} u_{2} \mathrm{~d} \Gamma-\int_{\partial \Omega_{B}} u_{2} \mathrm{~d} \Gamma}{\int_{\partial \Omega_{R}} u_{1} \mathrm{~d} \Gamma-\int_{\partial \Omega_{L}} u_{1} \mathrm{~d} \Gamma}
$$

In practice, using a finite element method, Eq. (A.9) becomes:

$$
v_{12}^{*}=-\frac{\frac{1}{N_{T}} \sum_{i=1}^{N_{T}} u_{2}^{i}-\frac{1}{N_{B}} \sum_{b}^{N_{B}} u_{2}^{i}}{\frac{1}{N_{R}} \sum_{i=1}^{N_{R}} u_{1}^{i}-\frac{1}{N_{L}} \sum_{i=1}^{N_{L}} u_{1}^{i}}
$$

where $N_{i}, i \in\{T, B, R, L\}$ are, respectively, the number of nodes on top, bottom, right and left edges.

\section{References}

1. Allaire, G.: Shape Optimization by the Homogenization Method. Springer, New York (2002). https://doi.org/10.1007/9781-4684-9286-6

2. Allaire, G., Dapogny, C., Delgado, G., Michailidis, G.: Multi-phase structural optimization via a level set method. ESAIM Control Optim. Calc. Var. 20(2), 576-611 (2014). https://doi.org/10.1051/cocv/2013076

3. Allaire, G., Jouve, F., Toader, A.: Structural optimization using sensitivity analysis and a level-set method. J. Comput. Phys. 194(1), 363-393 (2004). https://doi.org/10.1016/j.jcp.2003.09.032

4. Allaire, G., Pantz, O.: Structural optimization with FreeFem++. Struct. Multidiscip. Optim. 32(3), 173-181 (2006). https:// doi.org/10.1007/s00158-006-0017-y

5. Allais, L., Bornert, M., Bretheau, T., Caldemaison, D.: Experimental characterization of the local strain field in a heterogeneous elastoplastic material. Acta Metall. Mater. 42(11), 3865-3880 (1994). https://doi.org/10.1016/0956-7151(94)904529

6. Almgren, R.F.: An isotropic three-dimensional structure with Poisson's ratio = - 1. J. Elast. 15(4), 427-430 (1985). https:// doi.org/10.1007/BF00042531

7. Babaee, S., Shim, J., Weaver, J.C., Chen, E.R., Patel, N., Bertoldi, K.: 3D soft metamaterials with negative Poisson's ratio. Adv. Mater. 25(36), 5044-5049 (2013). https://doi.org/10.1002/adma.201301986

8. Bakhvalov, N., Panasenko, G.: Homogenisation: Averaging Processes in Periodic Media. Springer, Dordrecht (1989). https:// doi.org/10.1007/978-94-009-2247-1

9. Bendsøe, M.P., Sigmund, O.: Topology Optimization: Theory, Methods and Applications. Springer, Berlin (2004). https:// doi.org/10.1007/978-3-662-05086-6

10. Carraturo, M., Rocca, E., Bonetti, E., Hömberg, D., Reali, A., Auricchio, F.: Graded-material design based on phase-field and topology optimization. Comput. Mech. (2019). https://doi.org/10.1007/s00466-019-01736-w

11. Choi, J.B., Lakes, R.S.: Fracture toughness of re-entrant foam materials with a negative Poisson's ratio: experiment and analysis. Int. J. Fract. 80(1), 73-83 (1996). https://doi.org/10.1007/BF00036481

12. Clausen, A., Wang, F., Jensen, J.S., Sigmund, O., Lewis, J.A.: Topology optimized architectures with programmable Poisson's ratio over large deformations. Adv. Mater. 27(37), 5523-5527 (2015). https://doi.org/10.1002/adma.201502485

13. Duncan, O., Shepherd, T., Moroney, C., Foster, L., Venkatraman, P.D., Winwood, K., Allen, T., Alderson, A.: Review of auxetic materials for sports applications: expanding options in comfort and protection. Appl. Sci. 8(6), 941 (2018). https:// doi.org/10.3390/app8060941

14. Evans, K.E.: Auxetic polymers: a new range of materials. Endeavour 15(4), 170-174 (1991). https://doi.org/10.1016/01609327(91)90123-S

15. Friis, E.A., Lakes, R.S., Park, J.B.: Negative Poisson's ratio polymeric and metallic foams. J. Mater. Sci. 23(12), 4406-4414 (1988). https://doi.org/10.1007/bf00551939

16. Gao, J., Li, H., Luo, Z., Gao, L., Li, P.: Topology optimization of micro-structured materials featured with the specific mechanical properties. Int. J. Comput. Methods (2018). https://doi.org/10.1142/S021987621850144X

17. Greaves, G.N., Greer, A.L., Lakes, R.S., Rouxel, T.: Poisson's ratio and modern materials. Nat. Mater. 10(11), 823-837 (2011). https://doi.org/10.1038/nmat3134

18. Grima, J.N., Zammit, V., Gatt, R., Alderson, A., Evans, K.E.: Auxetic behaviour from rotating semi-rigid units. Phys. Status Solidi B 244(3), 866-882 (2007). https://doi.org/10.1002/pssb.200572706

19. Hecht, F.: New development in FreeFem++. J. Numer. Math. (2012). https://doi.org/10.1515/jnum-2012-0013

20. Hou, S., Liu, T., Zhang, Z., Han, X., Li, Q.: How does negative Poisson's ratio of foam filler affect crashworthiness? Mater. Des. 82, 247-259 (2015). https://doi.org/10.1016/j.matdes.2015.05.050

21. Imbalzano, G., Tran, P., Ngo, T.D., Lee, P.V.S.: A numerical study of auxetic composite panels under blast loadings. Compos. Struct. 135, 339-352 (2016). https://doi.org/10.1016/j.compstruct.2015.09.038 
22. Jantos, D.R., Riedel, C., Hackl, K., Junker, P.: Comparison of thermodynamic topology optimization with SIMP. Continu. Mech. Thermodyn. 31(2), 521-548 (2018). https://doi.org/10.1007/s00161-018-0706-y

23. Lakes, R.S.: Foam structures with a negative Poisson's ratio. Science 235(4792), 1038-1040 (1987). https://doi.org/10.1126/ science.235.4792.1038

24. Lakes, R.S.: Advances in negative Poisson's ratio materials. Adv. Mater. 5(4), 293-296 (1993). https://doi.org/10.1002/ adma.19930050416

25. Lakes, R.S., Elms, K.: Indentability of conventional and negative Poisson's ratio foams. J. Compos. Mater. 27(12), 1193-1202 (1993). https://doi.org/10.1177/002199839302701203

26. Larsen, U.D., Sigmund, O., Bouwsta, S.: Design and fabrication of compliant micromechanisms and structures with negative Poisson's ratio. J. Microelectromech. Syst. 6(2), 99-106 (1997). https://doi.org/10.1109/84.585787

27. Li, T., Chen, Y., Hu, X., Li, Y., Wang, L.: Exploiting negative Poisson's ratio to design 3D-printed composites with enhanced mechanical properties. Mater. Des. 142, 247-258 (2018). https://doi.org/10.1016/j.matdes.2018.01.034

28. Mei, C.C., Vernescu, B.: Homogenization Methods for Multiscale Mechanics. World Scientific, Singapore (2010). https:// doi.org/10.1142/7427

29. Milton, G.W.: Composite materials with poisson's ratios close to - 1. J. Mech. Phys. Solids 40(5), 1105-1137 (1992). https:// doi.org/10.1016/0022-5096(92)90063-8

30. Milton, G.W., Cherkaev, A.V.: Which elasticity tensors are realizable? J. Eng. Mater. Technol. 117(4), 483 (1995). https:// doi.org/10.1115/1.2804743

31. Nika, G., Constantinescu, A.: Design of multi-layer materials using inverse homogenization and a level set method. Comput. Methods Appl. Mech. Eng. 346, 388-409 (2019). https://doi.org/10.1016/j.cma.2018.11.029

32. Osher, S., Sethian, J.A.: Fronts propagating with curvature-dependent speed: algorithms based on Hamilton-Jacobi formulations. J. Comput. Phys. 79(1), 12-49 (1988). https://doi.org/10.1016/0021-9991(88)90002-2

33. Ranaivomiarana, N., Irisarri, F.X., Bettebghor, D., Desmorat, B.: Concurrent optimization of material spatial distribution and material anisotropy repartition for two-dimensional structures. Contin. Mech. Thermodyn. 31(1), 133-146 (2018). https:// doi.org/10.1007/s00161-018-0661-7

34. Réthoré, J., Leygue, A., Coret, M., Stainier, L., Verron, E.: Computational measurements of stress fields from digital images. Int. J. Numer. Methods Eng. 113(12), 1810-1826 (2017). https://doi.org/10.1002/nme.5721

35. Sanchez-Palencia, E., Zaoui, A.: Homogenization Techniques for Composite Media. Springer, Berlin (1987). https://doi.org/ 10.1007/3-540-17616-0

36. Saxena, K.K., Das, R., Calius, E.P.: Three decades of auxetics research-materials with negative Poisson's ratio: a review. Adv. Eng. Mater. 18(11), 1847-1870 (2016). https://doi.org/10.1002/adem.201600053

37. Sigmund, O.: Materials with prescribed constitutive parameters: an inverse homogenization problem. Int. J. Solids Struct. 31(17), 2313-2329 (1994). https://doi.org/10.1016/0020-7683(94)90154-6

38. Spadoni, A., Ruzzene, M., Gonella, S., Scarpa, F.: Phononic properties of hexagonal chiral lattices. Wave Motion 46(7), 435-450 (2009). https://doi.org/10.1016/j.wavemoti.2009.04.002

39. Ting, T.C.T., Chen, T.: Poisson's ratio for anisotropic elastic materials can have no bounds. Q. J. Mech. Appl. Math. 58(1), 73-82 (2005). https://doi.org/10.1093/qjmamj/hbh021

40. Vogiatzis, P., Chen, S., Wang, X., Li, T., Wang, L.: Topology optimization of multi-material negative Poisson's ratio metamaterials using a reconciled level set method. Comput. Aided Des. 83, 15-32 (2017). https://doi.org/10.1016/j.cad.2016.09. 009

41. Wang, F., Sigmund, O., Jensen, J.S.: Design of materials with prescribed nonlinear properties. J. Mech. Phys. Solids 69, 156-174 (2014). https://doi.org/10.1016/j.jmps.2014.05.003

42. Wang, X., Mei, Y., Wang, M.Y.: Level-set method for design of multi-phase elastic and thermoelastic materials. Int. J. Mech. Mater. Des. 1(3), 213-239 (2004). https://doi.org/10.1007/s10999-005-0221-8

43. Wang, Y., Luo, Z., Zhang, N., Kang, Z.: Topological shape optimization of microstructural metamaterials using a level set method. Comput. Mater. Sci. 87, 178-186 (2014). https://doi.org/10.1016/j.commatsci.2014.02.006

44. Zhang, H., Luo, Y., Kang, Z.: Bi-material microstructural design of chiral auxetic metamaterials using topology optimization. Compos. Struct. 195, 232-248 (2018). https://doi.org/10.1016/j.compstruct.2018.04.058 